\title{
Improved Frame Error Concealment Algorithm Based on Transform- Domain Mobile Audio Codec
}

\author{
Kai Xiang ${ }^{1,2,3}$, Shihong Chen ${ }^{1, *}$ and Yuanyuan Zeng ${ }^{2}$ \\ ${ }^{I}$ National Engineering Research Center for Multimedia Software, School of Computing, Wuhan University, Wuhan, \\ 430072, China \\ ${ }^{2}$ Su Zhou Institute of Wuhan University, Suzhou, 215123, China \\ ${ }^{3}$ School of Information Management, Hubei University of Economics, Wuhan, 430205, China
}

\begin{abstract}
In this paper, we propose an improved frame error concealment algorithm for TCX-based audio coders in order to improve the quality of decoded audio at the receiver. Conventional TCX-based frame error concealment algorithms in the AMR-WB+ audio codec are usually unable to adaptively adjust gain control according to changes in the magnitude of the correct frame before lost frames. Thus, we propose a new gain control approach based on stability of LPC filter, and the stream data need to contain the line spectrum frequency data and utilize different continuous attenuation factor formula based on different categories of lost frames. The proposed frame error concealment algorithm is designed for TCX-based audio codec. We make the performance comparison between the proposed method and the original algorithm currently employed in AMR-WB+ audio codec through the objective evaluation test and subjective evaluation test. The results prove that our algorithm can achieve better audio recovery quality than original frame error concealment algorithm employed in AMR-WB+ speech codec.
\end{abstract}

Keywords: Audio coding, TCX, frame error concealment, AMR-WB+.

\section{INTRODUCTION}

TCX(Transform Coded Excitation) performs encoding non-speech part for the hybrid speech coder, R.Salami and C.Lanamme et al., who first put forward TCX encoding method in 1993, and its application to music made better coding results in signal coding framework [1, 2]. In 1999, B.Bessette proposed the ACELP (Algebraic Code Excited Linear Prediction)/TCX hybrid coding framework, which can be implemented in a hybrid coder for speech and music signals to select an appropriate encoding mode according to the SNR of input signal [3]. In this paper, we introduce the TCX encoding method and select the TCX-1024 encoding mode. TCX encoding method utilizes the original mature LPC (Linear Predictive Coding) speech coding technology and the lattice vector quantization technology for spectral parameters, as shown in the following Fig. (1).

As shown in Fig. (1), TCX coding makes pre-processing the input signal sampling rate conversion process at first. And then it forms perceptual weighting filter through linear prediction. Then we get the combined signal from preprocessed signal by perceptual weighting filter processing. The combined signal changes to frequency domain through Discrete Fourier Transformation. The low frequency is shaped and followed by 8 -dimensional lattice vector quantization. We then get the gain of the generated signal and the original signal. The final output is spectral parameters (quantitative numbers), gain, ISF parameters and noise factor, and then the formation of bit stream output. Since TCX is a transform domain coding, the adaptive window with half drop cosine shape after the TCX frame is necessary for the smooth switch of ACELP and TCX mode. Decoder is based on the factors of the encoded output channel characteristic parameters of ISF, gain, noise factor and spectral parameters.

\section{THE ANALYSIS OF ERROR CONCEALMENT ALGORITHM IN THE TRANSFORM DOMAIN DE- CODER FOR AMR-WB+}

For AMR-WB+ error concealment in the transform domain, three models of TCX are used. We need to distinguish the pattern length. We set the frame length that received previously as "L1" and the lost fame length as "L2 after that. We use "L1-L2" to represent that in the following. And "L" as an abbreviation for the one-frame length.

1) "L-L" situation: In the scenarios of 256-256, 512-512 and 1024-1024, the frame length of the correctly received frame is equal to the lost frame. We can use the correctly received " $L$ " frames to reimburse the lost " $L$ " frames.

2) "L-L/2" situation: In the scenarios of 512-256 and 1024-512, the frame length of the correctly received frame is the twice as the lost frame. According to the correctly previous received frames with only the even number of data remained and the odd number of data ignored, we get that the 


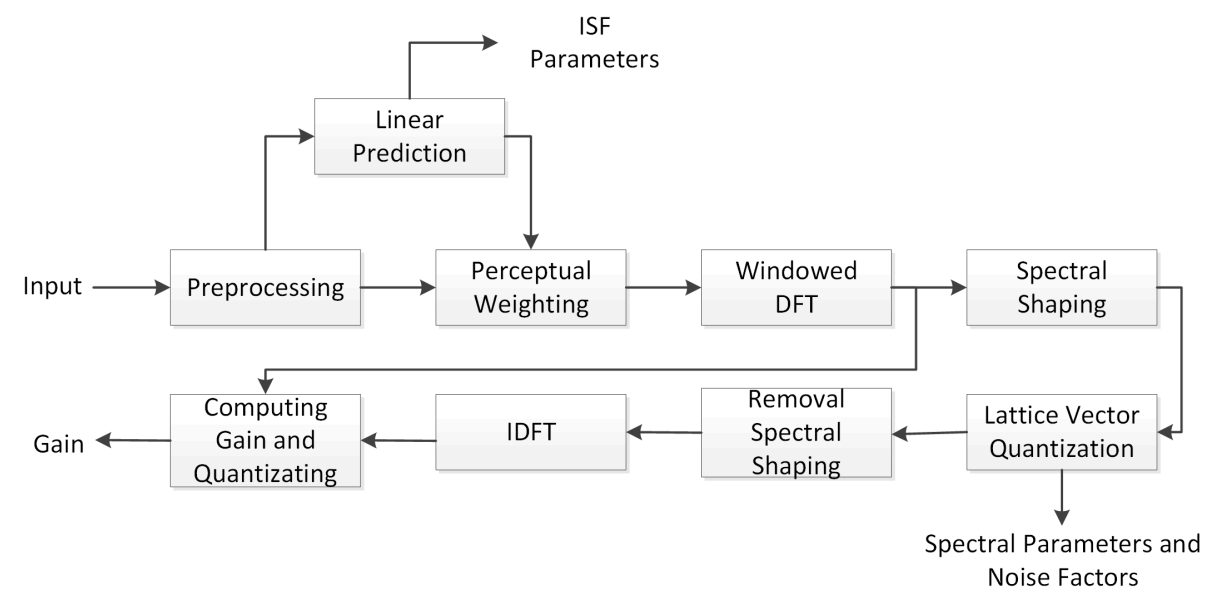

Fig. (1). TCX-coded scheme.

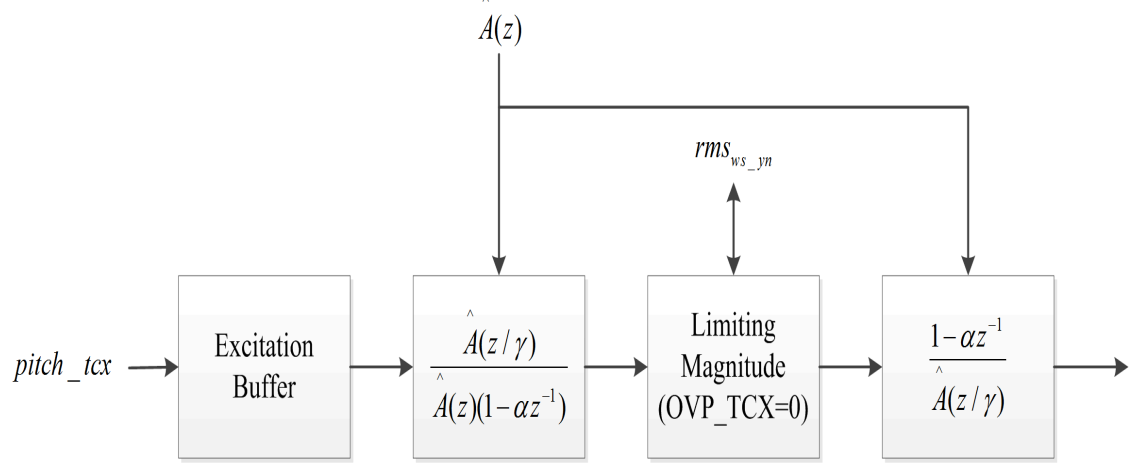

Fig. (2). Diagram of TCX-256 frame error concealment.

"L/2" length of data sequence can be the reimburses basis for the lost "L/2" data.

3) "L-2L" situation: In the scenarios of 256-512 and 5121024, the frame length of the previous received correct frames is the half of the lost frame after it. We insert a data unit of every two adjacent data units for the "L" frames. Then we get data sequence of " $2 \mathrm{~L}$ ", which is the reimburse basis for the lost frames. For the " $L$ " frames, the amplitude and phase of the two adjacent points are: $A_{1}, \varphi_{1}, A_{2}, \varphi_{2}$.

The amplitude and the phase of the data interpolation of the two points are shown in Equation (1).

$A=\left(A_{1}+A_{2}\right) / 2, \varphi=\left(\varphi_{1}+\varphi_{2}\right) / 2$

\subsection{Error Concealment for TCX-256}

In case of lost frames for TCX-256 mode, the decoder does not receive the valid information. The lost frame spectrum envelope is required to be obtained through reconstruction. The previous frame excited by delay with $T=p i t c h \_t c x$ and then produces the output signal through a nonlinear filter $1 / \hat{A}(z)$. The pitch_tcx is the pitch delay of the previous TCX frame decoded correctly.
As shown in Fig. (2), the nonlinear filter is with the transfer function as shown in Equation (2). After delay $T$, the excitation signals are mapped to the TCX frequency domain. The output signal is obtained after transmission of the amplitude restricted through function as in Equation (3).

$$
\begin{aligned}
& f(z)=\frac{A(z / \gamma)}{\hat{A}(z)} \times \frac{1}{1-\alpha z^{-1}} \\
& f(z)=\frac{1-\alpha z^{-1}}{\hat{A}(z / \gamma)}
\end{aligned}
$$

\subsection{Error Concealment in TCX-512 and TCX-1024}

In the following, we introduce the two models of TCX256 and TCX-1024 in details. Considering TCX-512 process is similar to TCX-1024, we do not discuss further in this paper again. AMR-WB+ for TCX-1024 mode, only two consecutive super frames with TCX-1024 pattern are processed, i.e., the local frame loss occurs of the current TCX-1024 super frame and the previous correct frame received is TCX1024 super frame. So the two consecutive TCX-1024 super 
frames are with Quasi-balanced states. The sub-vector of the lost frame can be obtained from the interpolation of the last frame. The lost frame recovery process is as follows:

1) Spectrum shaping: In the case of lost frames, the shaping process is to make evaluation of the new spectrum maximum by the quantization parameters of the previous frames. Since we need to calculate the block energy $E_{\max }$ in spectrum shaping, the outcome may be not accurate for lost spectrum. If $E_{\max }<$ old $E_{\max }$, then make $E_{\max }=$ old $E_{\max }$. Set $E_{\max }$ as the 8-dimension block energy of quantization index and then calculate the scale ratio parameter as in Equation (4):

$$
R_{m}=E_{\max } / E_{m}
$$

If $\left(R_{m}\right)^{1 / 2}>10$, and then set $R_{m}=10$, i.e., the maximal gain is $20 \mathrm{~dB}$. Otherwise, if $R_{m}>R_{m-1}$, then set $R_{m}=R_{m-1}$.

2) Spectrum extrapolation : Spectrum extrapolation is based on amplitude and phase separately. Extrapolation is based on amplitude and phase with certain continuity. Before the IFFT, the extrapolation of the quantized spectral parameters is executed.

a) Amplitude extrapolation: The amplitude extrapolation is executed according to the following steps:

Firstly, calculate the amplitude spectrum of previous frames as shown in Equation (5):

$$
\operatorname{old} A[k]=|\operatorname{old} \hat{X}[k]|
$$

Secondly, calculate the amplitude spectrum of the current frame as in Equation (6):

$$
A[k]=|\hat{X}[k]|
$$

Then, use the spectrum aptitude of the non-lost frame and the phase to the previous frames to calculate the amplitude gain as in Equation (7).

$$
\text { gain }=\sqrt{\frac{\sum_{k, S[k] \neq 0} A[k]^{2}}{\sum_{k, S[k] \neq 0} \text { old } A[k]^{2}}}
$$

From the calculated gain with the above equation, we then calculate the amplitude of the spectrum according to Equation (8).

$$
A[k]=\operatorname{gain} \times \operatorname{old} A[k]
$$

b) Phase extrapolation :

Firstly, we illustrate the packed way of data transmission. In the following, $[0,1,2,3]$ represents the first, the second, the third and the fourth data, and so on.

The original data as $[0,1,2,3,4,5,6,7,8,9, \ldots]$ is divided into four sub frames as:

Subframe_1: $\{[0,1,2,3],[16,17,18,19] \ldots\}$
Subframe_2: $\{[4,5,6,7],[20,21,22,23] \ldots\}$
Subframe_3: $\{[8,9,10,11],[24,25,26,27] \ldots\}$

Subframe_4: $\{[12,13,14,15],[28,29,30,31] \ldots\}$

Once the packet loss occurs, the entire frame is dropped. The same as phase interpolation, it uses the phase information of the former frames. In such cases, the part $K+1$, $K+N-1$ is missing. If $K, K+N$ is received correctly, the following process is needed to be done.

Assume $\varphi(K)$ and $\varphi(K+N)$ is known, the phase between them can be get according to the interpolation as shown in Equation(9).

$$
\hat{\varphi}(k)=\hat{\varphi}(k-1)+\Delta o l d \varphi(k)+\Delta \varphi_{c}, k=K+1, \ldots ., K+N-1
$$

Among it, we have Equation (10) and Equation (11).

$$
\begin{aligned}
& \Delta \operatorname{old} \varphi(k)=\operatorname{old} \varphi(k)-\operatorname{old} \varphi(k-1) \\
& \Delta \varphi_{c}=\frac{[\varphi(K+N)-\varphi(K)]-[\operatorname{old} \varphi(K+N)-\operatorname{old} \varphi(K)]}{N}
\end{aligned}
$$

Each lost data stream needs to calculate $\Delta \varphi_{c}$. For example, when lost Subframe_2, the section $[4,5,6,7]$ needs to calculate $\Delta \varphi_{c}$. The section $[20,21,22,23]$ needs to calculate $\Delta \varphi_{c}$ too.

The following three scenarios are to explain $\varphi(K)$ and $\varphi$ $(K+N)$ within the different conditions.

1) Regardless of the two ends: Between the two ends of a stream of error data can always receive data correctly. For example, if Subframe 1 is lost, $[16,17,18,19]$ is lost and [15], [20] can be received correctly, i.e., $\mathrm{K}+1, \mathrm{~K}+\mathrm{N}-1$ is lost but $\mathrm{K}$ and $\mathrm{K}+\mathrm{N}$ is received correctly. $\varphi(\mathrm{K})$ and $\varphi(\mathrm{K}+\mathrm{N})$ is the actual data.

2) Consider the lost part occurs at the front end of the frame, the lost data front end is not received correctly. In this case, Subframe_ 1 is definitely lost. The solution is as $\mathrm{K}=0$, and $\varphi(K)=\varphi(\overline{0})=0 . \varphi(K+N)$ is the actual value 。

3) Considering the lost part occurs at the end of the frame, the lost end data is not received correctly. In this case, Subframe_4 is definitely lost. The solution is $\Delta \varphi_{c}=0$.

From the above, AMR-WB+ in the transform domain for recovery of lost frames is executed according to frame type. As a hybrid codec, the decoder is based on the extrapolation results. For TCX-256, TCX-512 and TCX-1024, different error concealment methods are taken. However, AMR-WB+ error concealment method does not consider the oscillation of the lost frame. This may lead to the high distortion of recovery effects with the burst loss of last frames.

\section{TCX GAIN CONTROL METHOD BASED ON LPC FILTER STABILITY}

In view of the drawbacks of AMR-WB+ and the other encoder, we propose to use an adaptive gain control method based on the adaptive control of the original signal amplitude and power synthesis of lost frames. 


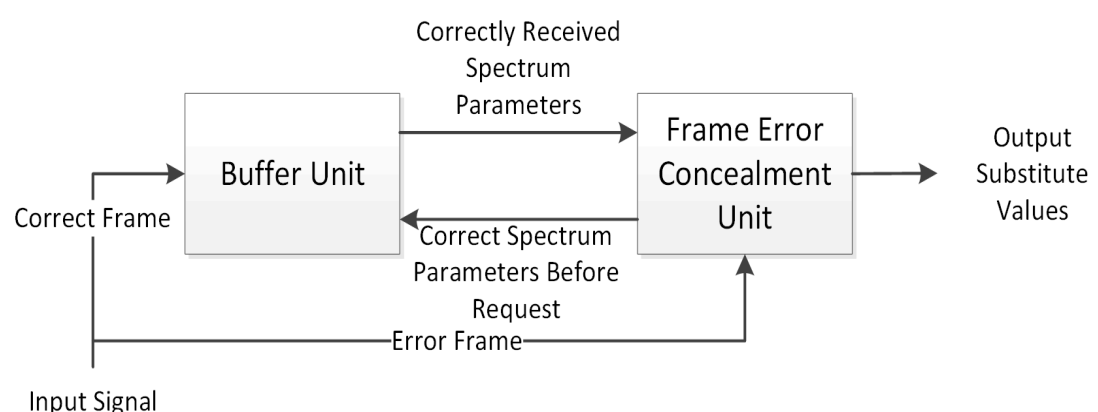

Fig. (3). Frame error concealment scheme.

Fig. (3) illustrates the recovery technology of the lost frame. It introduces a buffer unit on the decoding side. For the input bit stream signals, the information of the correct received frame is saved in buffer storage unit with a pre-set size that can be dynamically updated for accurate TCX frame data. In decoder, when the correct frames are received, the data is buffered in the unit. When the bad frame occurs, it triggered the error concealment generator. Then it applies to visit the correct data frames of the buffer, the spectrum coefficient substitutes of the bad frames are output according to the error concealment scheme.

TCX codec utilizes the linear prediction coding technology, i.e., to involve the linear filter parameters of the vocal tract model into LSF codec. So, we can evaluate the oscillation of the lost frames according to the linear filter stability. The linear filter stability is calculated according to the distance of the spectrum frequency as in Equation (12).

$$
L S F_{d i s t}=\sum_{i=0}^{u}\left(f_{i}-f_{i}^{(p)}\right)^{2}
$$

Among it, $u+1$ denotes the filter prediction parameter of the previous two correct received TCX frame. $f_{i}$ denotes the $i^{t h}$ filter parameter of the first correct received TCX frame. $f_{i}$ (p) denotes the $i^{\text {th }}$ filter parameter of the $2^{\text {nd }}$ correct received TCX frame. For the narrow-band signal, there are 10 filter prediction parameters. For the wide-band signal, there are 16 filter prediction parameters. On the codec side, the $k^{\text {th }}$ prediction filter parameter $f_{k}$ can be obtained from the autocorrelation of the input signal as shown in Equation (13).

$$
f_{k}=\sum_{n=k}^{t} s^{\prime}(n) s^{\prime}(n-k)
$$

Among it, $s^{\prime}(n)$ is a wide-band signal window. In the general codec, the Levinson-Durbin algorithm is been used a lot to calculate the prediction filter parameter. The prediction filter parameter is then transmitted to the decoder. Then we can calculate the filter stability according to the distance of the spectrum frequency. The stability metric $\theta$ is calculated as in Equation (14).

$$
\theta=1.25-L S F_{\text {dist }} / v
$$

We need to constrain the stability metric. When $\theta<0$, it set to value 0 . When $\theta>1$, it set to value 1 . The stability metric is among the range of $[0,1]$. Among it, $v$ is an integer.
When the input signal is the wide-band signal, $v$ is set to 400,000 . When the input signal is the narrow-band signal, $v$ is 156,250 . We then judge LPC filter stability according to the stability metric, i.e., when $\theta=1$ or $\theta \rightarrow 1$, the filter is very stabile. When $\theta=0$ or $\theta \rightarrow 0$, the filter is very unstable.

In the typical transform domain spectrum parameters recovery, the error concealment units usually use a gain metric multiply the corresponding point of the previous correct received frame to recover the spectrum information of the lost frames. For all frames, a fixed gain metric is utilized. Then we cannot distinguish the type of the lost frames and cannot refer the spectrum parameters discipline of the adjunct frames. In this paper, the attenuation factor $\varphi$ is used to represent the different attenuation degree of the different type. The attenuation factor $\varphi$ multiply the gain metric is to consider the frame type and information of the previous two frames. To involve stability $\theta$ into attenuation factor $\varphi$ calculation by using a simple linear relationship is based on the theory that the more unstable of the filter the more attenuation. A typical attenuation metric calculation is based on Equation (15).

$$
\varphi=0.65+0.2 \times \theta
$$

Lecomte Jérémie et al proposed some effective research work on attenuation parameter, i.e., considering the frame type and the number of the continuous lost frames [4]. It is effective to distinguish among the frame type, such as sonant transition type, the received frame includes periodic information with changes to the previous frame. For human initial type, the frame energy increases. From it, we can obtain the attenuation parameter table according to the frame type and the number of the consecutive lost frames as in Table $\mathbf{1}$.

Then we use spectrum extrapolation to calculate the amplitude gain as Equation (16), i.e., use the amplitude of the current non-lost frame and the phase to its previous frames.

gain $=\sqrt{\frac{\sum_{k, S[k] \neq 0} A[k]^{2}}{\sum_{k, S[k] \neq 0} \text { old } A[k]^{2}}}$

Among it, $A[K]$ represents the current amplitude, and old $A[K]$ represents the amplitude of the former correct frame. Then we calculate the gain metric gain according to Equation (16) and attenuation metric according to Equation 
Table 1. The attenuation parameter within different frame type and lost frame number.

\begin{tabular}{|c|c|c|}
\hline The Previous Correct Frame Received & The Number of Consecutive Lost Frames & Attenuation Parameters \\
\hline \hline Human initial & 0 & 0.6 \\
\hline Initial & $>3$ & $0.2 * \theta+0.8$ \\
\hline Initial & $>3$ & 0.4 \\
\hline Voiced transition & 0 & 0.8 \\
\hline Voiceless transition & $>1$ & $0.2 * \theta+0.75$ \\
\hline Voiceless transition & $=1$ & $0.2 * \theta+0.6$ \\
\hline Voiceless sound & $=2$ & $0.2 * \theta+0.4$ \\
\hline Voiceless sound & $>2$ & $0.2 * \theta+0.8$ \\
\hline Voiceless sound & $=1$ & $0.2 * \theta+0.65$ \\
\hline Voiced sound & $=2$ & $0.2 * \theta+0.5$ \\
\hline Voiced sound & $>2$ & 0.2 \\
\hline
\end{tabular}

(15). From this, we then calculate the spectrum value as Equation (17).

$$
X[k]=\varphi \times \text { gain } \times \text { old } X[k]
$$

\section{EXPERIMENT AND ANALYSIS}

In order to evaluate the performance of improved algorithm, this proposed algorithm is embedded in the AMR$\mathrm{WB}+$ codec. In this paper, experiments were designed to compare the filter stationary based conversion code validity excitation gain control method and its performance as compared to the algorithm of AMR-WB +. Therefore, in the event of lost frames, LSF distance is calculated according to the two preceding correctly received frame LSF coefficients and stability in order to determine the filter to obtain spectral parameters extrapolated gain control attenuation factor. In the experiment, a set of data includes a frame, in 4 random packet loss rate, the same set of test sequences were used to AMR-WB + decoder and decoding algorithm in this article is to improve the recovery sequence error concealment get lost frames after then use the weighted SNR method to determine the performance of the proposed algorithm and AMR-WB + algorithm.

In this paper, experimental devices for subjective listening tests are: DELL OptiPlex 380 desktops; sound card Intel 82801 GB ICH7; Philips headphones SHM6110Ux1. The hybrid encoder selects -rate 24 i.e. corresponding to the internal sampling rate $25.6 \mathrm{khz}, 12.4 \mathrm{~kb} / \mathrm{s}$ bit rate of AMR-WB + codec , the encoding mode, and the use of the three coding modes of TCX, the encoding and decoding are using mono of audio formats. Input sequences are using the MPEG standard TIMIT audio sequences library, select the sampling rate of $16 \mathrm{k}$ mono test sequence. 12 standard sequence contains three speech sequences and nine musical sequences, the name of the test sequences and the corresponding number shown in the following Table 2.
Table 2. 12 groups of test sequences.

\begin{tabular}{|c|c|}
\hline Sequence Number & Audio Sequences \\
\hline \hline 1 & es01.wav \\
\hline 3 & es02.wav \\
\hline 4 & es03.wav \\
\hline 5 & sc01.wav \\
\hline 6 & sc02.wav \\
\hline 7 & sc03.wav \\
\hline 8 & si01.wav \\
\hline 9 & si02.wav \\
\hline 10 & si03.wav \\
\hline 11 & sm01.wav \\
\hline 12 & sm02.wav \\
\hline & sm03.wav \\
\hline
\end{tabular}

In the subjective quality evaluation, we use BS.1534-1 subjective listening test method issued by ITU-R standard organization, which is one for the "medium quality " level signals a hidden reference and benchmark more excitation (MUSHRA), this method requires multiple listeners for listening tests, the results compared to an objective evaluation method better reflects the perceived effect of the human ears [5]. The audio quality of MUSHRA is divided into five levels from 0 to 100 , with each level range for 20 points, five levels were "Excellent", "Good", "Fair", "Poor" and "Bad". 


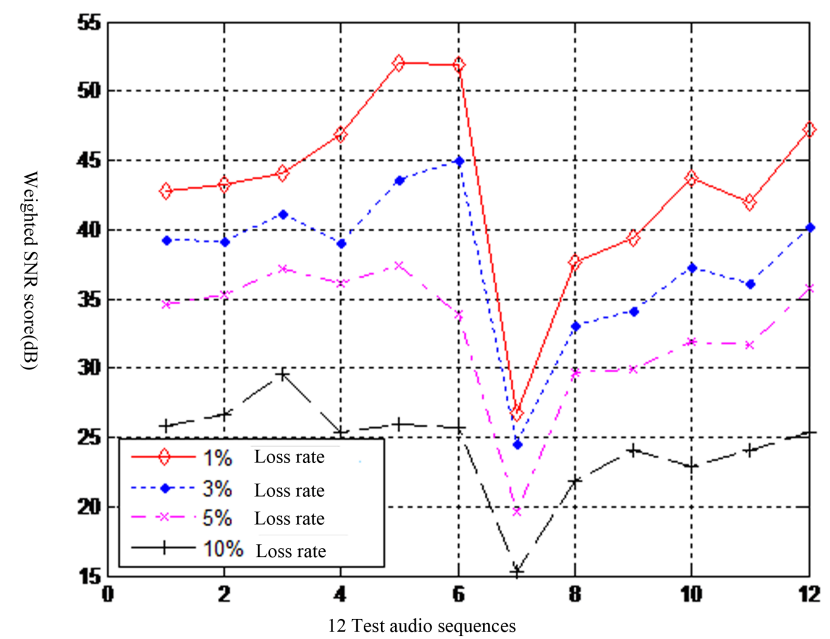

Fig. (4). Weighted SNR for AMR-WB + algorithm at four packet loss rates.

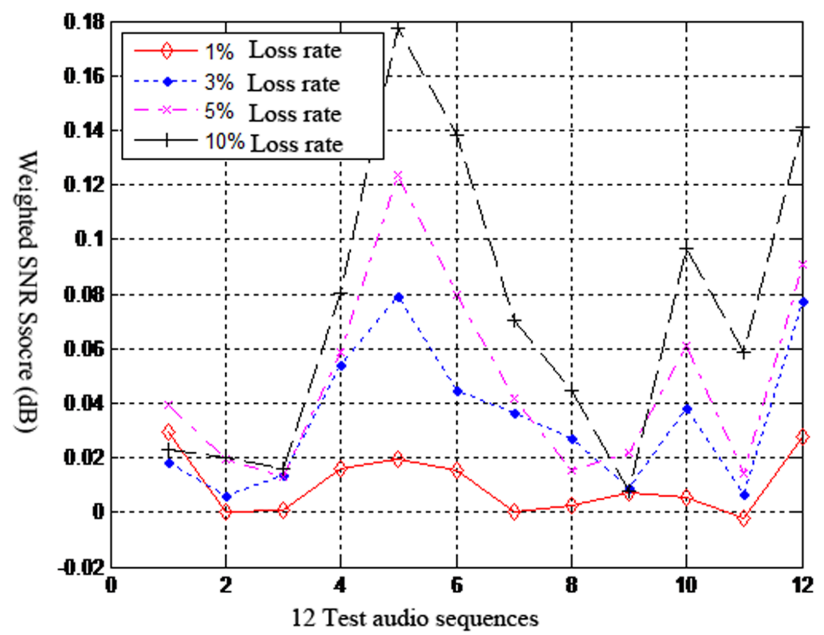

Fig. (5). Weighted SNR enhancement of proposed algorithm compared to the algorithm in AMR-WB+ at four packet loss rates.

Subjective acoustic effects is associated with listening environments, and listening devices also is directly related to the required selection criteria in listening tests. According to scoring statistics to evaluate the multiple listening quality of audio, this outcome should be excluded from the final dataprocessing part of extreme scores that certain extreme results or the results are of too much deviation from the average. Statistical results include the average score and standard deviation.

In the objective quality evaluation focuses on the objective evaluation of audio quality-weighted SNR. The calculation of weighted SNR requires testing for segments of reference vector and test vector, this method adopt the calculation formula for segments of the weighted SNR in AMR-WB +, due to the same importance of each frame, and so each frame's weights are the same.
In the frame loss concealment experiment, input fer.txt file for indicating the flag of lost frame which contains only the numbers 0 or 1 , every $20 \mathrm{~ms}$ basic frame corresponds to a number, $80 \mathrm{~ms}$ represents a super frame, 1 indicates the frame is lost, 0 indicates correct reception of the frame. It generate a random position in the file indicating a corresponding position for lost frames.

Experiments use the random packet loss rate, each contains one frame of data, and in the case of random packet loss it is generally not a consecutive packet loss situation at a lower loss rate. The Fig. (4) is the weighted SNR score for AMR-WB + algorithm and the proposed algorithm respectively at loss rate of $1 \%, 3 \%, 5 \%$ and $10 \%$.

As can be seen from the Fig. (5), the performance of proposed algorithm is relatively similar with the AMR-WB+ algorithm at lower packet loss rate, but when the packet loss 
Table 3. The weighted SNR score of algorithm compared with AMR-WB + algorithm.

\begin{tabular}{|c|c|c|c|}
\hline Loss Rate & AMR-WB+Algorithm(dB) & Proposed Algorithm(dB) & Promotion(dB) \\
\hline \hline $1 \%$ & 43.125 & 43.135 & 0.010 \\
\hline $3 \%$ & 37.705 & 37.740 & 0.035 \\
\hline $5 \%$ & 32.743 & 32.791 & 0.048 \\
\hline $10 \%$ & 24.390 & 24.462 & 0.072 \\
\hline AVG & 34.491 & 34.532 & 0.041 \\
\hline
\end{tabular}

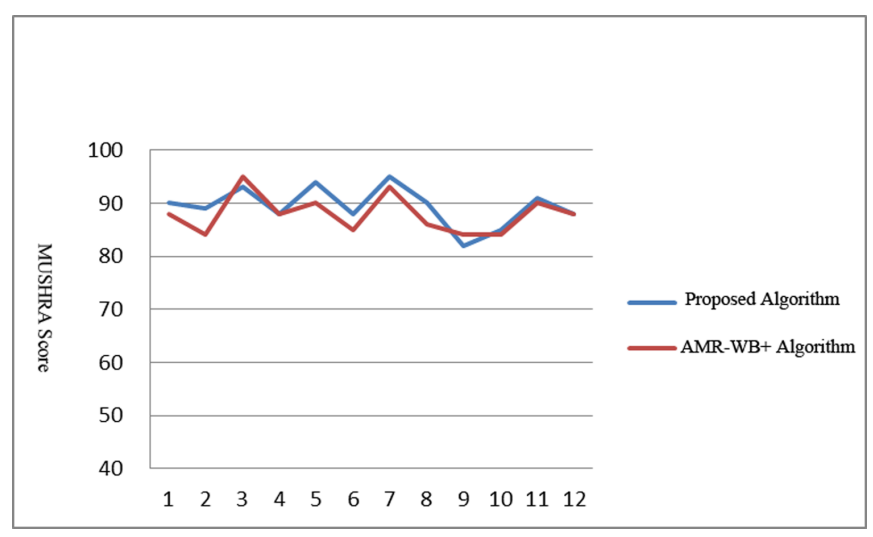

(a) At loss rate of $1 \%$

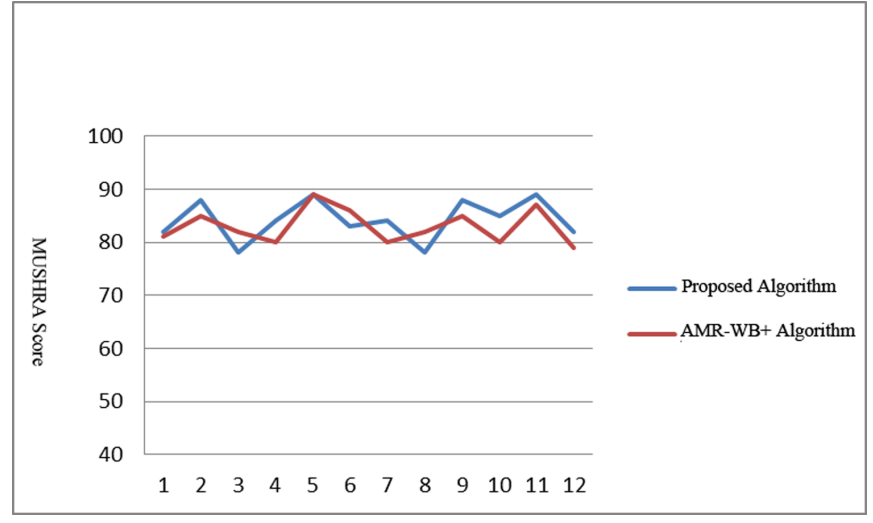

(b) At loss rate of $3 \%$

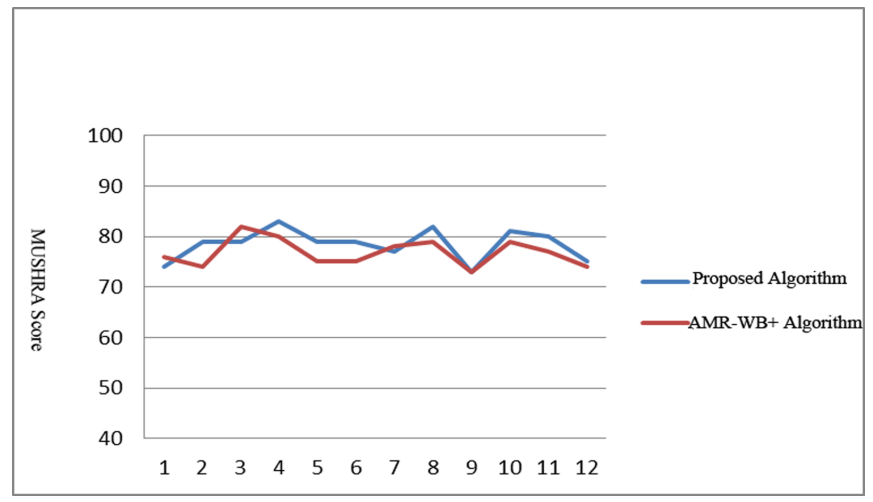

(c) At loss rate of 5\%

Fig. (6). Contd... 


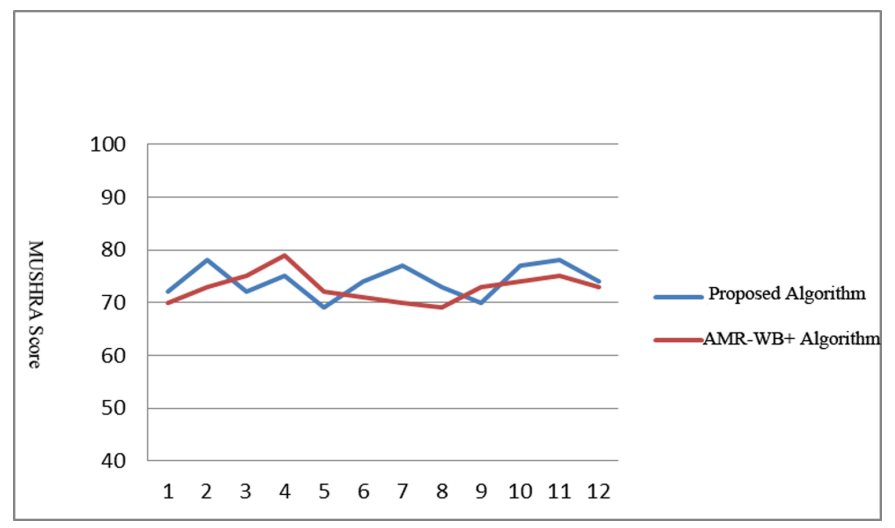

(d) At loss rate of $10 \%$

Fig. (6). Subjective scores of proposed algorithm compared with AMR-WB + algorithm at four random packet loss rates.

rate increases to $10 \%$, compared to the AMR-WB + algorithm, the proposed algorithm has significantly improved the value of weighted SNR up to $0.18 \mathrm{~dB}$. And the algorithm has amplitude variation gain control, so the greater music sequences has better enhancement effect than the speech sequence.

Details on the Table 3 is shown that the comparison between proposed algorithm and AMR-WB + algorithm under four kinds of random packet loss rate of twelve groups of test sequences. As the greater the loss rate, the higher performance could be obtained by proposed algorithm.

All twelve groups of MPEG test sequences at four random packet loss rate were respectively recovered frame loss concealment algorithm by AMR-WB + algorithm and proposed algorithm, and the decoded audio sequences were scored by MUSHRA scoring software, reference sequences were chosen from none-lost frames and correctly decoded sequences, we selected eight experienced listeners and twelve MPEG standard double-blind listening test sequences , the following scoring results can be obtained by averaging the results $\mathrm{s}$ of the eight listeners in the subjective test.

As can be seen from the Fig. (6), with the rise of packet loss rate, the subjective listening test scores will drop slightly, as subjective scores goes very high at $1 \%$ loss rate and that is considered that the restore lost frame signal and the original frame are almost the same; while loss rate goes up to $10 \%$, it comes up to the most significant decline, but still score than 70 points and prove that the occasionally missing a frame does not significantly reduce signal quality for auditory perception of the reconstructed audio. At the four kinds of random packet loss rate, the proposed algorithm has obtained better average scores of each test audio sequence compared with the AMR-WB + codec.

\section{CONCLUSION}

In this paper, we introduce the basic coding and decoding methods of AMR-WB+ codec using transform coded excitation. And then we analyze the error concealment with AMR$\mathrm{WB}+$ codec within the situation of frame loss, which includes the spectrum shaping removal, and spectrum extrapolation as well as phase extrapolation. Since original algorithm of AMR-WB+ cannot adjust the gain control adaptive- ly according to the accurate frame change amplitude before frame loss, we propose a gain control method of LPC filter with stability. We use LSF distance as the metric for LPC filter stability evaluation, i.e., the LSF distance on each dimension of the two accurate frames before frame loss. Then the stability metric is achieved by attenuation factor for gain control through linear transformation. The more instable of the filter, the fading is bigger. In our method, we propose to include LSF data within the code stream data, and then choose different attenuation factors according to different numbers and types of frame loss. The experiments are implemented by comparing with the original AMR-WB+ algorithms to testify the performance of error concealment at $1 \%$, $3 \%, 5 \%$, and $10 \%$ random packet loss rate. The experiments are held with twelve MPEG testing sequences. The results show our algorithms improve the additive signal SNR and MUSHRA objective listening results are also improved.

The experiments results show the effectiveness of our algorithm.

\section{CONFLICT OF INTEREST}

The author confirms that this article content has no conflict of interest.

\section{ACKNOWLEDGEMENTS}

This work was supported by the Major National Science and Technology Special Projects (2010ZX03004-003-03), National Nature Science Foundation of China (No. 61231015, No. 61102127, No. 61201340, No.61201169, No.61272278), the Natural Science Foundation of Jiangsu Province Youth Project (No. BK2012200), Foundation of Department of Education of Hubei Province (No. Q20132207).

\section{REFERENCES}

[1] R. Lefebvre, R. Salami, C. Laflamme, J.-P.Adoul, "8kbit/s coding of speech with 6ms frame length," IEEE International Conference on Acoustics, Speech, and Signal Processing(ICASSP),Mineasota,USA, pp.612-615, Apr.1993.

[2] R. Lefebvre, R. Salami, C. Laflamme JP. Adoul, "High quality coding of wideband audio signals using transform coded excitation (TCX)", IEEE International Conference on Acoustics,Speech and 
Signal Processing(ICASSP), Adelaide, Australia, pp.1193-1196, Apr.1994.

[3] B. Bessette, R. Salami, C. Laflamme, R. Lefebvre, "A wideband speech and audio codec at 16/24/32kbit/s using hybrid ACELP/TCX techniques," IEEE Workshop on Speech Coding, Porvoo, Finland, pp. 7-9, Jun.1999.
[4] L. Jérémie, D. Martin, S. Michael, S. Ralph, "Apparatus and Method for Error Concealment in Low-delay Unified Speech and Audio Coding(USAC)," WIPO Patent Application WO/2012/110447 Kind Code: A1, Aug.2012.

[5] ITU-R: Recommendation BS.1534-1, "Method for the subjective assessment of intermediate quality levels of coding systems," International Telecommunication Union, 2003.

Received: October 16, 2014

(C) Xiang et al.; Licensee Bentham Open.

This is an open access article licensed under the terms of the Creative Commons Attribution Non-Commercial License (http://creativecommons.org/licenses/by$\mathrm{nc} / 3.0 /$ ) which permits unrestricted, non-commercial use, distribution and reproduction in any medium, provided the work is properly cited. 